\title{
Nonspecific Organelle-Targeting Strategy with Core-Shell Nanoparticles of Varied Lipid Components/Ratios
}

\author{
Lu Zhang, ${ }^{\dagger}$ Jiashu Sun, ${ }^{*}+$ Yilian Wang, ${ }^{\S}$ Jiancheng Wang, ${ }^{\S}$ Xinghua Shi, ${ }^{\ddagger}$ and Guoqing Hu ${ }^{\|}$ \\ ${ }^{\dagger}$ Department of Chemistry, Capital Normal University, Beijing 100048, China \\ ${ }^{\ddagger}$ CAS Key Laboratory for Biological Effects of Nanomaterials and Nanosafety, CAS Center for Excellence in Nanoscience, National \\ Center for Nanoscience and Technology, Beijing 100190, China \\ ${ }^{\S}$ State Key Laboratory of Natural and Biomimetic Drugs, School of Pharmaceutical Sciences, Peking University, Beijing 100191, \\ China \\ "State Key Laboratory of Nonlinear Mechanics, Institute of Mechanics, Chinese Academy of Sciences, Beijing 100190, China
}

Supporting Information

ABSTRACT: We report a nonspecific organelle-targeting strategy through one-step microfluidic fabrication and screening of a library of surface charge- and lipid components/ratiosvaried lipid shell-polymer core nanoparticles. Different from the common strategy relying on the use of organelle-targeted moieties conjugated onto the surface of nanoparticles, here, we program the distribution of hybrid nanoparticles in lysosomes or mitochondria by tuning the lipid components/ratios in shell. Hybrid nanoparticles with 60\% 1,2-dioleoyl-3-trimethylammonium-propane (DOTAP) and 20\% 1,2-dioleoyl-sn-glycero-3-
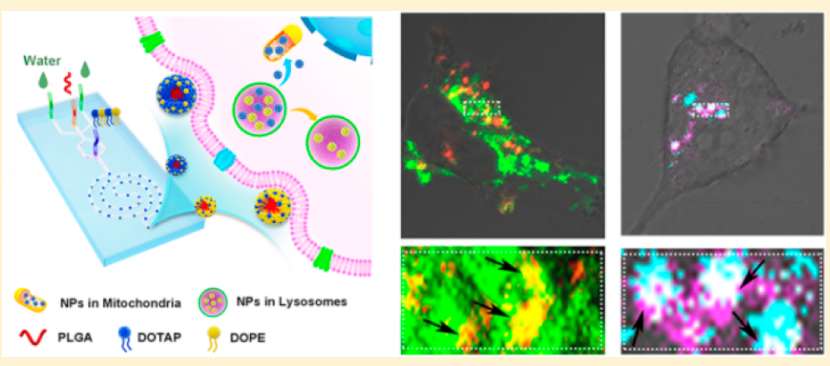
phosphoethanolamine (DOPE) can intracellularly target mitochondria in both in vitro and in vivo models. While replacing DOPE with the same amount of 1,2-dipalmitoyl-sn-glycero-3-phosphocholine (DPPC), the nanoparticles do not show mitochondrial targeting, indicating an incremental effect of cationic and fusogenic lipids on lysosomal escape which is further studied by molecular dynamics simulations. This work unveils the lipid-regulated subcellular distribution of hybrid nanoparticles in which target moieties and complex synthetic steps are avoided.

I ntracellular transport of nanocarriers is one of the key steps in drug delivery. To sharply increase the therapeutic efficiency, drug-loaded nanoparticles need to be intracellularly delivered to specific organelles, such as mitochondria or lysosomes. $^{1-3}$ For example, the primary action site of many anticancer drugs is the mitochondrial membrane. ${ }^{4}$ The lysosomal targeting of enzymes is of primary importance for the therapy of lysosomal storage diseases. ${ }^{5-7}$ To modulate the distribution of nanoparticles in mitochondria or lysosomes, the conventional strategies are to conjugate the surface of nanoparticles with organelle-targeting compounds. For example, triphenylphosphonium, ${ }^{8,9}$ peptide, ${ }^{10}$ and octaarginine ${ }^{11}$ have been linked to nanoparticles for targeting mitochondria, ${ }^{12}$ while nanoparticles modified with octadecyl-rhodamine $\mathrm{B}^{13}$ or amphiphilic guanidinylated neomycin ${ }^{14}$ can target lysosomes. However, the surface modification of nanoparticles always involves complex synthetic steps, large batch-to-batch variations, and a wide size distribution of generated nanoparticles. ${ }^{15}$ Moreover, the conjugated targeting moieties may affect the stability and cellular uptake, as well as in vivo circulation of nanoparticles. $^{16-20}$

Among a variety of pharmaceutical carriers, lipid-polymer hybrid nanoparticles have received a lot of attention for gene delivery and cancer therapy. ${ }^{21,22}$ In comparison with soft liposomes of a bilayer membrane structure, the rigid lipid shell-polymer core nanoparticles exhibit enhanced cellular uptake, high therapeutic efficacy, and long circulation half-time, in which the solid polymer core encapsulates drugs and the lipid shell improves the biocompatibility. ${ }^{23-25}$ The cellular uptake of lipid-polymer nanoparticles depends on their physiochemical properties, including sizes, shapes, surface charges, surface modifications, and mechanical properties. ${ }^{26-30}$

However, few efforts have been devoted to investigate their subcellular distribution. This might be due to most of the hybrid nanoparticles entering the cell via the endocytic pathway, resulting in the entrapment of nanoparticles in endosomes and eventually to lysosomes for degradation. ${ }^{31,32}$ To date, the design of organelle-targeted hybrid nanoparticles without the use of targeting moieties poses a huge challenge.

In this work, we propose a new approach to modulate the subcellular distribution of lipid shell-poly(lactide-co-glycolide) (PLGA) core nanoparticles by changing the lipid components/ ratios, instead of complex conjugation of targeting molecules. A library of lipid-polymer nanoparticles with varied surface properties is fabricated inside a microfluidic device, which

Received: May 3, 2016

Accepted: June 17, 2016

Published: June 17, 2016 

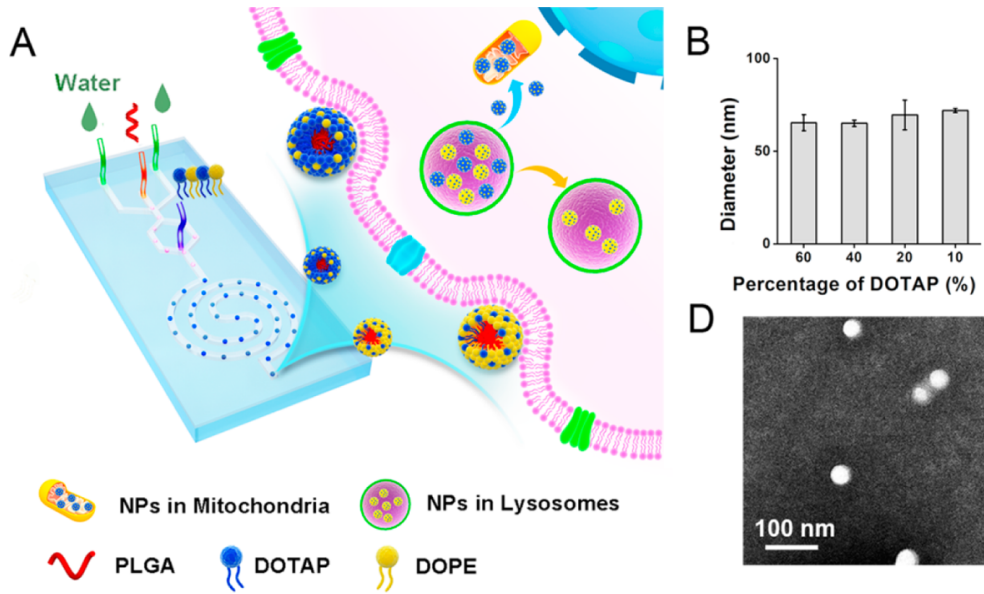

C

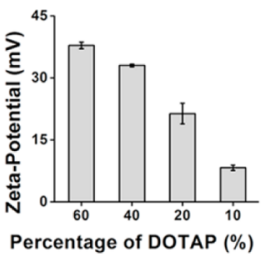

$\mathrm{D}$
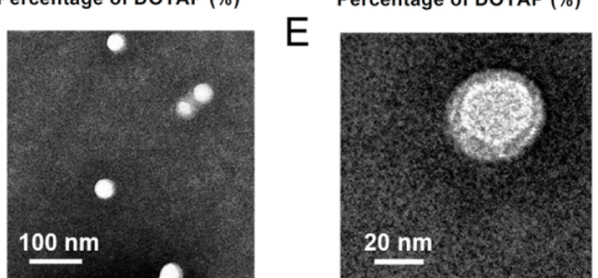

Figure 1. (A) Schematic of the microfluidic chip for fabrication of PLGA-lipid core-shell nanoparticles (NPs) with different lipid components/ ratios. By adjusting the DOTAP/DOPE ratio from 3 to 0.14 in lipid shell, hybrid nanoparticles intracellularly target mitochondria or lysosomes. DLS measurement of (B) hydrodynamic diameter and (C) zeta potential of hybrid nanoparticles. The percentage of DOTAP varies from $60 \%$ to $10 \%$ and that of DOPE is from $20 \%$ to $70 \%$. The percentages of cholesterol and DSPE-PEG in lipid shell are $16 \%$ and $4 \%$, respectively. (D, E) TEM images of hybrid nanoparticles with PLGA-core, lipid-shell structures.

allows for the layer-by-layer assembly and high-throughput screening of hybrid nanoparticles. ${ }^{33-37}$ The subcellular distribution of lipid-polymer nanoparticles is thoroughly studied by in vitro and in vivo experiments. The underlying mechanism of lipid-regulated subcellular distribution of hybrid nanoparticles is elucidated experimentally and numerically, which may advance the understanding of organelle-specific drug delivery or imaging in nanomedicine.

\section{EXPERIMENTAL SECTION}

Materials. Poly(D,L-lactide-co-glycolide) (PLGA, lactide/ glycolide $=75: 25$ ) was purchased from SurModics. 1,2Dioleoyl-3-trimethylammonium-propane (DOTAP), 1,2-dioleoyl-sn-glycero-3-phosphoethanolamine (DOPE), 1,2-dipalmitoyl-sn-glycero-3-phosphocholine (DPPC), cholesterol, and 1,2-distearoyl-sn-glycero-3-phosphoethanolamine- $N$-[methoxy(polyethylene glycol)]-2000 (DSPE-PEG2000) were purchased from Avanti. Phosphotungstic acid, ethanol, dimethylformamide (DMF), trifluoroethanol (TFE), and dimethyl sulfoxide (DMSO) were purchased from Sigma. DiD, Mitotracker Green FM, and Lysotracker Yellow HCK-123 were purchased from Invitrogen. Dulbecco's modified Eagle's medium (DMEM), trypsin, fetal bovine serum, penicillin/streptomycin, and $10 \times$ phosphate buffered saline (PBS) were purchased from Gibco.

Design and Fabrication of the Microfluidic Device. The microfluidic device for generation of lipid-polymer nanoparticles with different lipid components/ratios consists of two stages with four inlets and one outlet (Figures $1 \mathrm{~A}$ and S1): (1) the first stage is composed of three inlet microchannels connected to a straight microchannel for precipitation of a polymer core of hybrid nanoparticles; (2) the second stage is composed of one middle inlet and a double spiral microchannel for self-assembling of lipid shell onto polymer core to form hybrid nanoparticles, which are collected from the outlet. The inlet channels in the first stage are $100 \mu \mathrm{m}$ in width and $50 \mu \mathrm{m}$ in depth. The other microchannels are $300 \mu \mathrm{m}$ in width and 50 $\mu \mathrm{m}$ in depth. More details of microfluidic chip design can be found in the previous publication. ${ }^{26,38-40}$

On-Chip Generation of Lipid-Polymer Nanoparticles. The lipid-polymer core-shell nanoparticles with different lipid components/ratios were continuously generated inside the microfluidic device by the following protocols. PLGA solution (1\%) and water were injected into the middle and side inlets of the first stage of the microfluidic device at 3 and $240 \mathrm{~mL} / \mathrm{h}$ $(120 \mathrm{~mL} / \mathrm{h}$ for each side inlet) using the syringe pumps (PHD Ultra, Harvard Apparatus). Lipid solution (2 $\mathrm{mM}$ ) was simultaneously injected into the middle inlet of the second stage at $3 \mathrm{~mL} / \mathrm{h}$. PLGA solution (1\%) was prepared by dissolving the PLGA powder in the mixture of TFE and DMF $(3: 7, \mathrm{v} / \mathrm{v})$. Lipid solution $(2 \mathrm{mM})$ contained 4\% DSPE-PEG, $16 \%$ cholesterol, 60\%-10\% DOTAP, and 20\%-70\% DOPE (or 20\% DPPC) dissolved in ethanol (Table S1). The PLGA core of hybrid nanoparticles was precipitated in the first stage, and the lipid shell was assembled onto the PLGA core via hydrophobic interaction, which results in the lipid-shell PLGAcore nanoparticles. For preparing DiD-labeled nanoparticles, $1 \%$ PLGA solution containing $10 \mu \mathrm{M} \mathrm{DiD}$ was used.

Characterization of Lipid-Polymer Nanoparticles. Dynamic light scattering (DLS, Zetasizer 3000HS, Malvern Instruments Ltd.) was used to measure the hydrodynamic diameter and zeta potential of lipid-polymer nanoparticles with different lipid components/ratios. For size measurement, $40 \mu \mathrm{L}$ of nanoparticle suspension in a mini-DLS cuvette was placed in the sample holder and analyzed by backscatter optics light at an angle of $173^{\circ}$. For measurement of zeta potential, $700 \mu \mathrm{L}$ of nanoparticle suspension in a disposable folded capillary cell was analyzed by DLS. At least triplicate measurements at $25{ }^{\circ} \mathrm{C}$ were performed on each sample. When the zeta potential is larger than $\pm 30 \mathrm{mV}$, the colloidal solution is considered as stable.

The structure of lipid-polymer nanoparticles was observed under transmission electron microscopy (TEM, FEI Tecnai T20). Carbon-coated copper grids were covered with $10 \mu \mathrm{L}$ of nanoparticle suspension for $5 \mathrm{~min}$ followed by negative staining with phosphotungstic acid for $10 \mathrm{~min}$. Excess stain was carefully removed using filter paper, and the copper grids were air-dried for $12 \mathrm{~h}$. The dried grids with attached lipid-polymer nanoparticles were observed at an accelerating voltage of 200 $\mathrm{kV}$ by TEM.

Cell Culture and Cellular Uptake. Human umbilical vein endothelial cells (HUVEC) were cultured in Dulbecco's modified Eagle's medium (DMEM) supplemented with 1\% 
penicillin/streptomycin and $10 \%$ fetal bovine serum (FBS) at $37{ }^{\circ} \mathrm{C}$ in the cell incubator $\left(5 \% \mathrm{CO}_{2}\right.$, Thermo Scientific). For cellular uptake analysis, HUVEC cells were seeded at a density of $1 \times 10^{5}$ cells per dish in confocal culture dishes and grown for $24 \mathrm{~h}$ for attachment. The cell culture medium was then changed to fresh DMEM medium without FBS but containing different concentrations of DiD-labeled lipid-polymer nanoparticles. The final concentration of PLGA from hybrid nanoparticles in the medium was $12.5,25$, or $50 \mu \mathrm{g} / \mathrm{mL}$. After incubation for $24 \mathrm{~h}$, HUVEC cells were observed using a laser scanning confocal microscope (LSM710, Zeiss). The fluorescent intensity of HUVEC cells was analyzed with ImageJ software (NIH, version 1.49).

The fluorescence intensity from DiD-labeled nanoparticles in HUVEC cells increases by 8.5 times with the increased amount of nanoparticles (PLGA concentration in medium: 12.5-25 $\mu \mathrm{g} / \mathrm{mL}$ ), indicating a tendency of enhanced cellular uptake (Figure S2). By further increasing the amount of nanoparticles (PLGA: $50 \mu \mathrm{g} / \mathrm{mL}$ ), the fluorescence intensity in HUVEC cells is similar to the previous case (PLGA: $25 \mu \mathrm{g} / \mathrm{mL}$ ), and the cellular uptake of nanoparticles by HUVEC cells is saturated (Figure S2). We thus choose the hybrid nanoparticles containing $25 \mu \mathrm{g} / \mathrm{mL}$ of PLGA for the following investigation.

Transmembrane Mechanism Study. Chlorpromazine (CPZ), genistein, and ethylisopropyl amiloride (EIPA) were used to inhibit the clathrin-mediated endocytosis, caveolaemediated endocytosis, and macropinocytosis, respectively. HUVEC cells were seeded in the culture dishes and grown for $24 \mathrm{~h}$ for attachment. Each of the three endocytosis inhibitors was then added into the dish with the final concentration of $30 \mu \mathrm{M}$ (CPZ), $200 \mu \mathrm{M}$ (Genistein), and 20 $\mu \mathrm{M}$ (EIPA). After $1 \mathrm{~h}$ of incubation, fresh culture medium containing the DiD-labeled nanoparticles with $10 \%$ or $60 \%$ DOTAP replaced the medium containing endocytosis inhibitors. The untreated cells were used as control. Cells were incubated with nanoparticles for $2 \mathrm{~h}$ and then washed 3 times with PBS. The fluorescence of nanoparticles in cells was observed by a LSM710 confocal microscope (Zeiss) with excitation wavelength of $633 \mathrm{~nm}$. Image $(\mathrm{NIH})$ was used to quantify the fluorescence in cells.

Colocalization Analysis of Lipid-Polymer Nanoparticle. HUVEC cells were cultured in confocal culture dishes and coincubated with lipid-polymer nanoparticles with different lipid components/ratios. The incubation condition was the same as the aforementioned protocol. The final concentration of PLGA from hybrid nanoparticles in the incubation medium was $25 \mu \mathrm{g} / \mathrm{mL}$. After $24 \mathrm{~h}$ of incubation, HUVEC cells were stained with $300 \mathrm{nM}$ Lysotracker or 500 $\mathrm{nM}$ Mitotracker for $30 \mathrm{~min}$ and then washed with PBS. The stained cells were observed with the laser scanning confocal microscope (LSM710, Zeiss). The excited wavelengths were $488 \mathrm{~nm}$ for Mitotracker or Lysotracker and $633 \mathrm{~nm}$ for DiDlabeled lipid-polymer nanoparticles.

Co-localization analysis of lipid-polymer nanoparticles with Mitotracker or Lysotracker inside HUVEC cells was performed with ImageJ colocalization finder plugin. Pearson's correlation coefficient $(R)$ describing the degree of overlap between two images was calculated by the following formula: ${ }^{41}$

$$
R=\frac{\sum_{i}\left(\mathrm{Sg}_{i}-\mathrm{Sg}_{\mathrm{avg}}\right)\left(\mathrm{Sr}_{i}-\mathrm{Sr}_{\mathrm{ave}}\right)}{\sqrt{\sum_{i}\left(\mathrm{Sg}_{i}-\mathrm{Sg}_{\mathrm{ave}}\right)^{2} \sum_{i}\left(\mathrm{Sr}_{i}-\mathrm{Sr}_{\mathrm{ave}}\right)^{2}}}
$$

in which Sg is the fluorescent intensity of pixels in the green (or cyan) channel focusing on stained organelles and $\mathrm{Sr}$ is the fluorescent intensity of pixels in the red (or magenta) channel focusing on DiD-labeled nanoparticles. $\mathrm{Sg}_{\text {ave }}$ and $\mathrm{Sr}_{\text {ave }}$ are the average intensities of these channels.

Biocompatibility Evaluation. Biocompatibility of nanoparticles was evaluated by using the sulforhodamine B (SRB) assay. HUVEC cells were seeded in a 96-well culture plate at a density of $3 \times 10^{3}$ cells per well and grown overnight. Nanoparticle solution with various concentrations was added in the 96-well plate. Cells without treatment were used as control. After 24 or $48 \mathrm{~h}$ of incubation, the cell viability was determined by using the SRB assay with detection at an absorbance of 490 nm using a microplate reader (Tecan Infinite M200).

In Vivo Distribution of Lipid-Polymer Nanoparticles. Female BALB/c nude mice $(18-20 \mathrm{~g})$ were purchased from Beijing HFK bioscience Co., LTD (Beijing, China) and raised in a specific pathogen free (SPF) environment. All care and handling of animals were performed with the approval of Institutional Authority for Laboratory Animal Care of Institute of Process Engineering, Chinese Academy of Science. MCF-7 cells $\left(5 \times 10^{6}\right)$ were inoculated subcutaneously (s.c.) in the back of the Balb/c nude mice. When tumor size reached $\sim 100$ $\mathrm{mm}^{3}$ in volume (volume $=0.5 \times$ length $\times$ width), the mice were administrated with $100 \mu \mathrm{L}$ of nanoparticles (PLGA concentration: $625 \mu \mathrm{g} / \mathrm{kg}$ ) through intra tumor injection. After $24 \mathrm{~h}$, the mice were sacrificed and the tumor tissues were harvested and immediately placed in Tissue-Tek OCT embedding medium (Sakura Finetek, Tokyo, Japan), frozen at $-80{ }^{\circ} \mathrm{C}$, and cut into $20 \mu \mathrm{m}$ cryosections. The sections were incubated with $1 \%$ BSA to block unspecific interactions for $2 \mathrm{~h}$ at room temperature and then with 1:200 anti-COX-IV mouse monoclonal antibody (Abcam, Cambridge, UK) overnight at 4 ${ }^{\circ} \mathrm{C}$ to label mitochondria. After washing with Tris buffered saline (TBS), sections were incubated with a 1:400 dilution of FITC-conjugated goat antimouse IgG (Invitrogen, Carlsbad, USA) at $37^{\circ} \mathrm{C}$ for $1 \mathrm{~h}$. The sections were then washed with TBS for 3 times and mounted with DAPI containing mounting medium (Abcam, Cambridge, UK). Sections were visualized with a confocal laser scanning microscope (LSM710, Zeiss).

Statistical Analysis. The differences between the experimental groups and control group were analyzed by one way analysis of variance (ANOVA) followed by Dunnett's Multiple Comparison Test. Statistical analysis was performed with the Graphpad prism software (Version 5.0.1, Graphpad software Inc.). A value of $P<0.05$ was considered as statistically significant.

\section{RESULTS AND DISCUSSION}

Fabrication and Characterization of Lipid-PLGA Nanoparticles. To fabricate hybrid nanoparticles with varied lipid components/ratios in one-step, we use a two-stage microfluidic chip (Figures 1A and S1). In the first stage, 1\% PLGA solution and two water sheaths are simultaneously injected into the straight synthesis channel from the middle and side inlets at the flow rate ratio of 80 (water, $240 \mathrm{~mL} / \mathrm{h}$; PLGA solution, $3 \mathrm{~mL} / \mathrm{h}$ ). Under a high flow rate ratio, the PLGA core of nanoparticles is first precipitated by hydrodynamic focusing. In the second stage, $2 \mathrm{mM}$ lipid solution containing varied ratios of DOTAP, DOPE or DPPC, cholesterol, and DSPEPEG is introduced through the center inlet (Table S1). The concentrations of DSPE-PEG and cholesterol in $2 \mathrm{mM}$ lipid solution keep constant as $4 \%$ and $16 \%$. The amounts of 


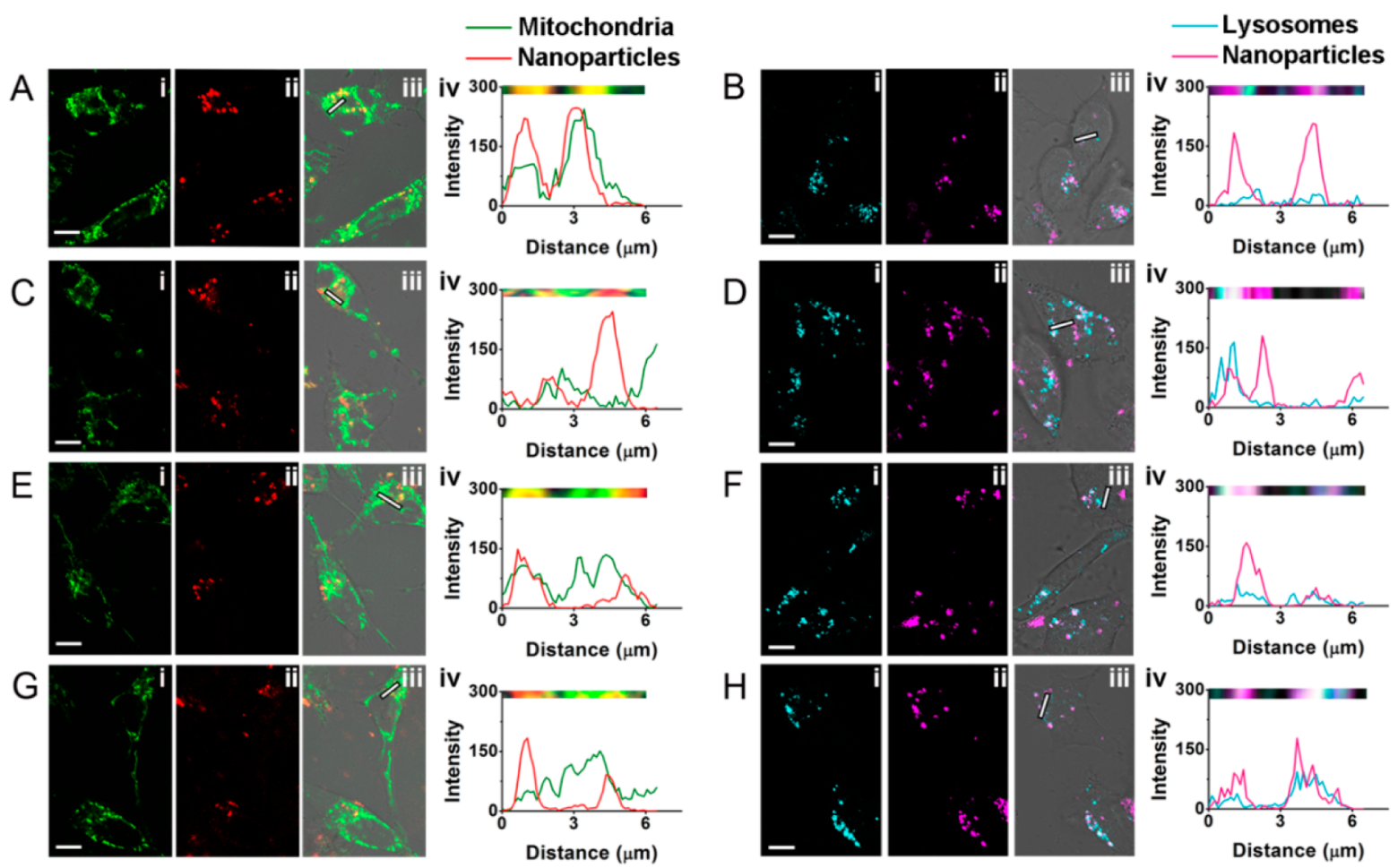

Figure 2. Images of HUVEC cells incubated with lipid-polymer nanoparticles containing (A, B) 60\% DOTAP and 20\% DOPE, (C, D) 40\% DOTAP and 40\% DOPE, (E, F) 20\% DOTAP and 60\% DOPE, and (G, H) 10\% DOTAP and 70\% DOPE in lipid shell. (i) Images of the green fluorescent channel indicating the stained mitochondria (A, C, E, G). Images of the cyan fluorescent channel indicating the stained lysosomes in (B, D, F, H). (ii) Images of the red fluorescent channel (A, C, E, G) and magenta fluorescent channel (B, D, F, H) indicating the DiD-labeled nanoparticles. (iii) Overlaid images of fluorescent channel and bright field. (iv) Fluorescence intensity profiles indicate the degree of overlap between hybrid nanoparticles and mitochondria/lysosomes. Inserted: magnified images in (iii) that are highlighted by short white lines. Scale bar, $10 \mu \mathrm{m}$.

DOTAP are $60 \%, 40 \%, 20 \%$, and $10 \%$, while those of DOPE are $20 \%, 40 \%, 60 \%$, and $70 \%$. The ratio of DOTAP/DOPE varies from 3 to 0.14 in lipid shell. Within the double spiral microchannel at the second stage, lipids of a monolayer structure are absorbed onto the surface of PLGA core through hydrophobic interaction. The generated core-shell lipidpolymer nanoparticles are continuously collected from the outlet.

After fabrication of lipid-polymer nanoparticles by microfluidic chip, we use transmission electron microscopy (TEM) and dynamic light scattering (DLS) to characterize their size, structure, and surface charge. TEM observation clearly shows the core-shell structure of hybrid nanoparticles (Figure 1E). Despite the varied DOTAP/DOPE ratios in lipid shell, the hydrodynamic diameters of hybrid nanoparticles are similar $(\sim 70 \mathrm{~nm}$, Figure 1B). We also use DLS to measure the surface charge of hybrid nanoparticles. As expected, the zeta potential of nanoparticles decreases from 37.9 to $8.3 \mathrm{mV}$ with the decreased amount of cationic lipid DOTAP from $60 \%$ to $10 \%$ (Figure 1C).

Subcellular Distribution of Hybrid Nanoparticles. Subcellular distribution of hybrid nanoparticles with varied DOTAP/DOPE ratios in lipid shell is studied by comparing the colocalization of nanoparticles with lysosomes/mitochondria. The optimized concentration of hybrid nanoparticles uptaken by HUVEC cells is determined to be $25 \mu \mathrm{g} / \mathrm{mL}$ PLGA in medium (Figure S2). After $24 \mathrm{~h}$ of coculture of DiD-labeled hybrid nanoparticles (red/magenta fluorescence, PLGA concentration: $25 \mu \mathrm{g} / \mathrm{mL}$; lipid concentration: $4.39 \mu \mathrm{M}$ ) and HUVEC cells, we use the organelle-specific dyes, Mitotracker (green fluorescence) and Lysotracker (cyan fluorescence), to label the mitochondria and lysosomes in HUVEC cells, respectively. Overlaying the confocal microscopic images from green and red fluorescent channels indicates a high level of colocalization for mitochondria and DiD-labeled hybrid nanoparticles with $60 \%$ DOTAP, which is further confirmed by the line scanning profiles (Figure $2 \mathrm{~A}$ ). For nanoparticles with $40 \%$ or $20 \%$ DOTAP in lipid shell, a wide distribution of nanoparticles in both mitochondria and lysosomes is observed (Figure 2C-F). With the decreased amount of DOTAP to $10 \%$, a colocalization for lysosomes and nanoparticles is detected by overlaid images from cyan and magenta fluorescent channels, as well as the corresponding line scanning profiles (Figure 2H).

We next use Pearson's correlation coefficient $(R)$ to quantitatively determine the colocalization of mitochondria/ lysosomes and hybrid nanoparticles (Figures 3 and S3). ${ }^{42} R$ is one of the standard measurements in colocalization analysis, by describing the correlation of intensity distributions of each pixel between channels. The value of $R$ is between -1.0 and 1.0, in which 1.0 indicates strong correlation and -1.0 indicates complete negative correlation. Quantitative analysis implies that mitochondrial targeting ability of hybrid nanoparticles is improved with increasing amount of DOTAP from $10 \%$ to $60 \%$ in lipid shell, indicated by the increased $R$ value from 0.21 \pm 0.14 to $0.40 \pm 0.17$ (Figure 3A). Meanwhile, the colocalization of nanoparticles with lysosomes decreases with the increased amount of DOTAP and reaches a maximum $R$ value of $0.62 \pm 0.14$ for $10 \%$ DOTAP (Figure 3B). These investigations reveal that the distribution of hybrid nanoparticles in mitochondria and lysosomes dramatically depends on the DOTAP/DOPE ratio in lipid shell. 
A

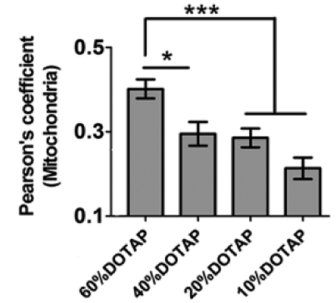

B

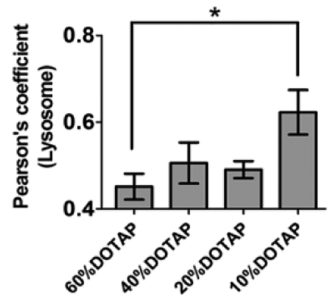

C。
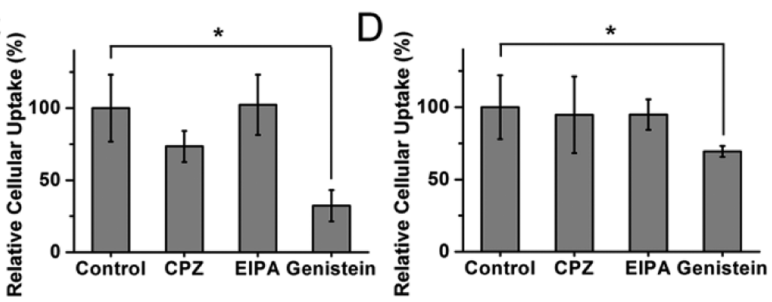

Figure 3. Calculated Pearson's correlation coefficient $(R)$ indicates the colocalization of (A) mitochondria or (B) lysosomes with hybrid nanoparticles of different amounts of DOTAP in lipid shell. (C) Effects of different endocytosis inhibitors on cellular internalization of hybrid nanoparticles with (C) $60 \%$ or (D) $10 \%$ DOTAP. * $P \leq 0.05$; $* * P \leq 0.01 ; * * * P \leq 0.001$.

Intracellular Trafficking of Lipid-Polymer Nanoparticles. The nonspecific subcellular distribution of hybrid nanoparticles is correlated with their intracellular trafficking. The intracellular process of hybrid nanoparticles can be simplified as three steps: (1) the cross of cell membrane to be internalized by cells, (2) the translocation within endo/ lysosomes, and (3) the endo/lysosomal escape of nanoparticles before being uptaken by other organelles. ${ }^{43}$ To study the internalization pathway of hybrid nanoparticles, HUVEC cells are preincubated with different endocytosis inhibitors (CPZ for inhibition of clathrin-mediated endocytosis, EIPA for inhibition of macropinocytosis, and Genistein for inhibition of caveolaemediated endocytosis). Genistein treatment decreases the intracellular uptake of hybrid nanoparticles with $60 \%$ or $10 \%$ DOTAP by $68 \%$ or $31 \%$, respectively, revealing that caveolaemediated endocytosis is the primary uptake mechanism for hybrid nanoparticles (Figure 3C,D). After internalization by endocytosis pathways, the intracellular trafficking of hybrid nanoparticles is studied by a time-dependent colocalization observation (Figure 4A,B). For both hybrid nanoparticles, colocalization with lysosomes is observed after incubation with HUVEC cells for $1 \mathrm{~h}$. After $24 \mathrm{~h}$ of incubation, hybrid nanoparticles with $60 \%$ DOTAP show a high level of colocalization with mitochondria, whereas hybrid nanoparticles with 10\% DOTAP are accumulated inside lysosomes. The highly efficient endo/lysosomal escape of hybrid nanoparticles with $60 \%$ DOTAP is partially attributed to the disruptive interaction between endosomal membranes and the cationic lipid shell of nanoparticles. ${ }^{31}$ The escaped nanoparticles of positive surface charge could be further attracted by mitochondria of the highly negative membrane potential. ${ }^{1}$
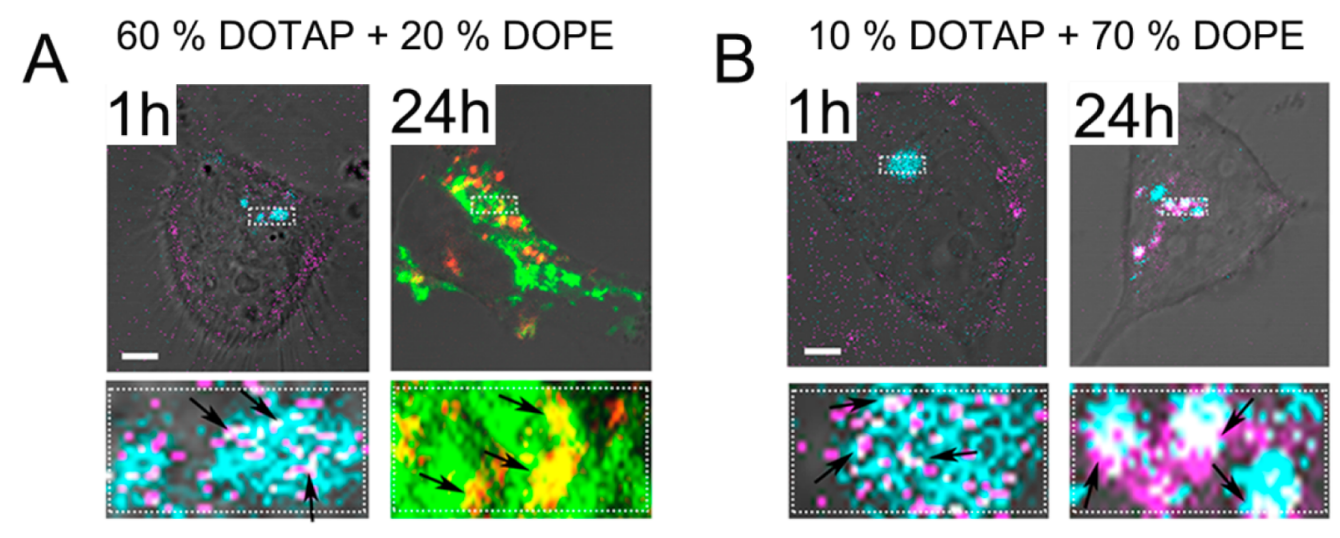

$60 \%$ DOTAP + 20 \% DPPC @ 24h

$80 \%$ DOTAP @ 24h
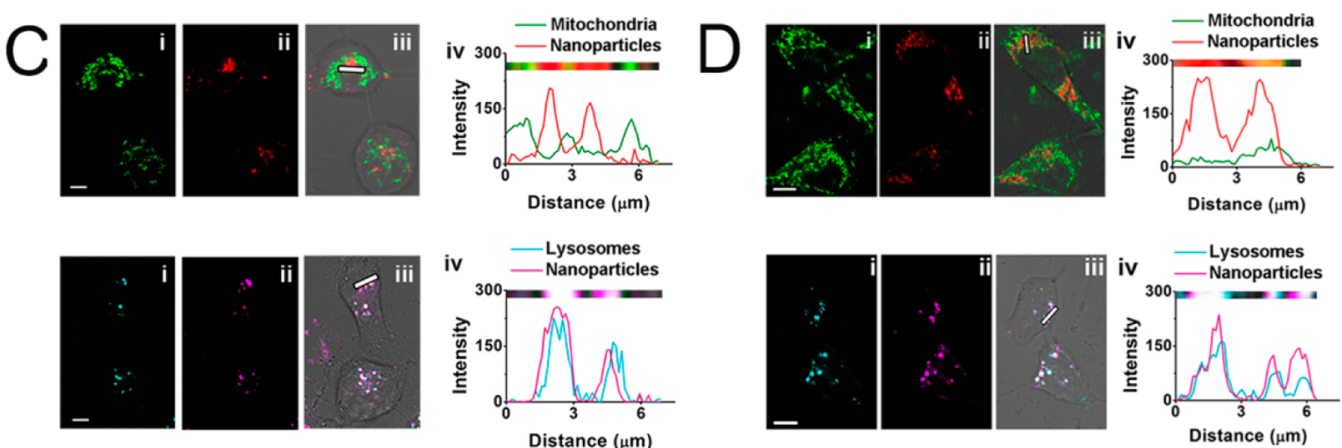

Figure 4. Confocal microscopic images of HUVEC cells incubated with lipid-polymer nanoparticles containing (A) 60\% DOTAP and 20\% DOPE and (B) $10 \%$ DOTAP and 70\% DOPE for 1 and $24 \mathrm{~h}$. The green fluorescent channel indicates the stained mitochondria, the red/magenta fluorescent channel indicates DiD-labeled nanoparticles, and the cyan fluorescent channel indicates the stained lysosomes. The black arrows in zoomed images indicate the colocalization of nanoparticles with stained organelles. Images of HUVEC cells incubated with lipid-polymer nanoparticles containing (C) 60\% DOTAP and 20\% DPPC and (D) $80 \%$ DOTAP for $24 \mathrm{~h}$. Fluorescence intensity profiles in (iv) indicate the degree of overlap between hybrid nanoparticles and mitochondria/lysosomes. Scale bar, $5 \mu \mathrm{m}$. 
Role of Lipids in Subcellular Distribution. In addition to the positive surface charge that affects the subcellular distribution of hybrid nanoparticles, we next investigate the roles of different lipid components/ratios in lipid shell. Hybrid nanoparticles containing 60\% DOTAP, 20\% DPPC, $16 \%$ cholesterol, and 4\% DSPE-PEG are fabricated by the same microfluidic device. DPPC is a neutral but nonfusogenic lipid of similar chemical structure with DOPE. ${ }^{44}$ The hybrid nanoparticles with 60\% DOTAP and 20\% DPPC show similar diameter $(68.9 \pm 1.6 \mathrm{~nm})$ and zeta potential $(36.1 \pm 1.2 \mathrm{mV})$, in comparison with nanoparticles containing 60\% DOTAP and $20 \%$ DOPE $(65.3 \pm 4.3 \mathrm{~nm}$ in size and zeta potential of $37.9 \pm$ $0.8 \mathrm{mV})$. To measure subcellular distribution of hybrid nanoparticles with 60\% DOTAP and 20\% DPPC, we coculture DiD-labeled nanoparticles with HUVEC cells for $24 \mathrm{~h}$ and observe the fluorescence with a confocal microscope. The images reveal that only few nanoparticles with 60\% DOTAP and $20 \%$ DPPC are colocalized with the mitochondria (Figure $4 \mathrm{C}, R=0.13 \pm 0.22$ ), while most of them are colocalized with the lysosomes (Figure 4C, $R=0.60 \pm 0.16$ ). This observation is opposite to nanoparticles with 60\% DOTAP and 20\% DOPE that exhibit a mitochondrial-targeted distribution (Figure 4A). We thus deduce that the absence of DOPE in lipid shell of hybrid nanoparticles may lead to the entrapment of nanoparticles in endo/lysosomes, preventing the relocalization of nanoparticles into mitochondria.

We also study the sole effect of DOTAP or DOPE by fabricating hybrid nanoparticles with $80 \%$ DOTAP $(72.0 \pm 1.2$ $\mathrm{nm}$ in size, and $39.5 \pm 1.9 \mathrm{mV}$ in zeta potential) or nanoparticles with $80 \%$ DOPE $(46.3 \pm 3.7 \mathrm{~nm}$ in size, and $-18.3 \pm 0.4 \mathrm{mV}$ in zeta potential). Without the presence of DOPE, a high level of colocalization for lysosomes and DiDlabeled hybrid nanoparticles with $80 \%$ DOTAP is observed after culturing nanoparticles with HUVEC cells for $24 \mathrm{~h}$ (Figure 4D). The $R$ values that quantify the colocalization of nanoparticles (80\% DOTAP) with lysosomes and mitochondria are determined as $0.66 \pm 0.15$ and $0.20 \pm 0.10$, respectively, indicating a lysosomal-specific distribution. As for the DiDlabeled nanoparticles with $80 \%$ DOPE, we cannot observe their fluorescent signals in HUVEC cells after a $24 \mathrm{~h}$ incubation, possibly due to the low cellular uptake of negatively charged nanoparticles (Figure S4). These investigations elucidate that the organelle-targeted distribution of hybrid nanoparticles is an incremental effect of cationic (DOTAP) and neutral helper (DOPE) lipids.

MD Simulations of Fusion of Nanoparticles with Lysosomal Membrane. It is commonly speculated that the lysosomal escape of lipids-coated nanoparticles is via the fusion of lipids with the membrane of lysosomes. A previous study indicates that neutral helper lipid DOPE could form an inverted hexagonal phase to increase the fusion of liposomal membrane. ${ }^{45}$ Our experimental study also confirms an incremental effect of DOTAP and DOPE on efficiently promoting the lysosomal escape of hybrid nanoparticles. To mimic this fusion process, the coarse grained (CG) molecular dynamics (MD) simulations are adopted with Martini force field to describe the interaction potentials. ${ }^{46} \mathrm{We}$ have constructed one nanoparticle with 3800 DOPE and DOTAP lipids (with ratio of about $1: 3$, shown in white and green in Figure 5A,B) and a patch of bilayer with 8200 DPPC lipids (shown in blue in Figure 5A,B). To accelerate the simulation, we use implicit solvent in this model. The bilayer plane lies parallel to the $x y$-plane, and the nanoparticle is arranged in
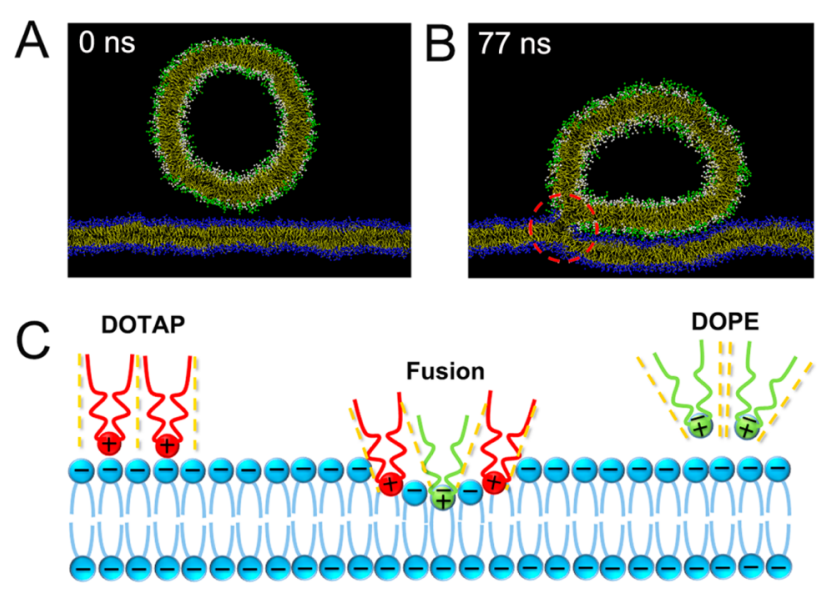

Figure 5. Molecular dynamics simulations to show the fusion of nanoparticles (composed of DOPE and DOTAP with ratio of about 1:3) with lysosomal membrane. (A) The nanoparticle is placed above the bilayer plane (at a distance of around $0.8 \mathrm{~nm}$ ) at $0 \mathrm{~ns}$. (B) The fusion occurs at the contact edge between the nanoparticle and bilayer at $77 \mathrm{~ns}$, indicated by a red dashed circle. The white, green, and blue colors represent the heads of DOPE, DOTAP, and DPPC. (C) Illustration of an incremental effect of DOTAP and DOPE to promote the fusion of lysosomal membrane.

close proximity above the bilayer (at a distance of around 0.8 $\mathrm{nm}$ ). A periodic boundary condition is applied in all three dimensions. The simulations are conducted in an isothermalisobaric (NPT) ensemble at a temperature of $323 \mathrm{~K}$ and a pressure of 0 bar. The Berendsen barostat with semi-isotropic pressure coupling is used to adjust the pressure. The nonbonded interaction is calculated with a cutoff of $1.2 \mathrm{~nm}$. In simulations, the time step is $40 \mathrm{fs}$. We note that we have alternatively changed the time step to $20 \mathrm{fs}$ and obtained similar results. All simulations are performed with the GROMACS software package, and VMD was used for molecular visualization. It is seen that, as the nanoparticle adhering to the bilayer, the fusion first occurs at the contact edge between the nanoparticle and bilayer, which is thought to have high curvature. The lipids around the high curvature regime are unstable and prone to fuse with the bilayer (Figure 5B). We note that, during the fusion, the contact and adhesion of nanoparticle onto bilayer is the first step for the bilayer to hold the nanoparticle, which is facilitated by the positive charge of DOTAP head. While in the fusion process, the role of DOPE lipid is important, which is typically thought to help to create the hydration core due to its unsaturated tails (Figure 5C).

Biocompatibility and in Vivo Mitochondrial Targeting. The biocompatibility of hybrid nanoparticles is evaluated by coculturing HUVEC cells with nanoparticles containing $60 \%$ or $10 \%$ DOTAP. After coincubation for $48 \mathrm{~h}$, hybrid nanoparticles with the lipid concentration of $4.39 \mu \mathrm{M}$ (the same concentration for a subcellular distribution experiment) or less do not affect the cell viability, regardless of different DOTAP/DOPE ratios in lipid shell (Figure 6A,B). In addition to in vitro investigation, we also evaluate in vivo mitochondrial distribution of lipid-polymer nanoparticles. We inject nanoparticles (60\% DOTAP, 20\% DOPE, 16\% cholesterol, and $4 \%$ DSPE-PEG in lipid shell) directly into the tumor tissue constituted by MCF-7 cells. After 24 h, we harvest the tumor tissue and cut the tumor tissue into cryosections. By comparing the fluorescence from DiD-labeled nanoparticles (red fluorescence, Figure 6D) and immune-stained mitochondria (green 

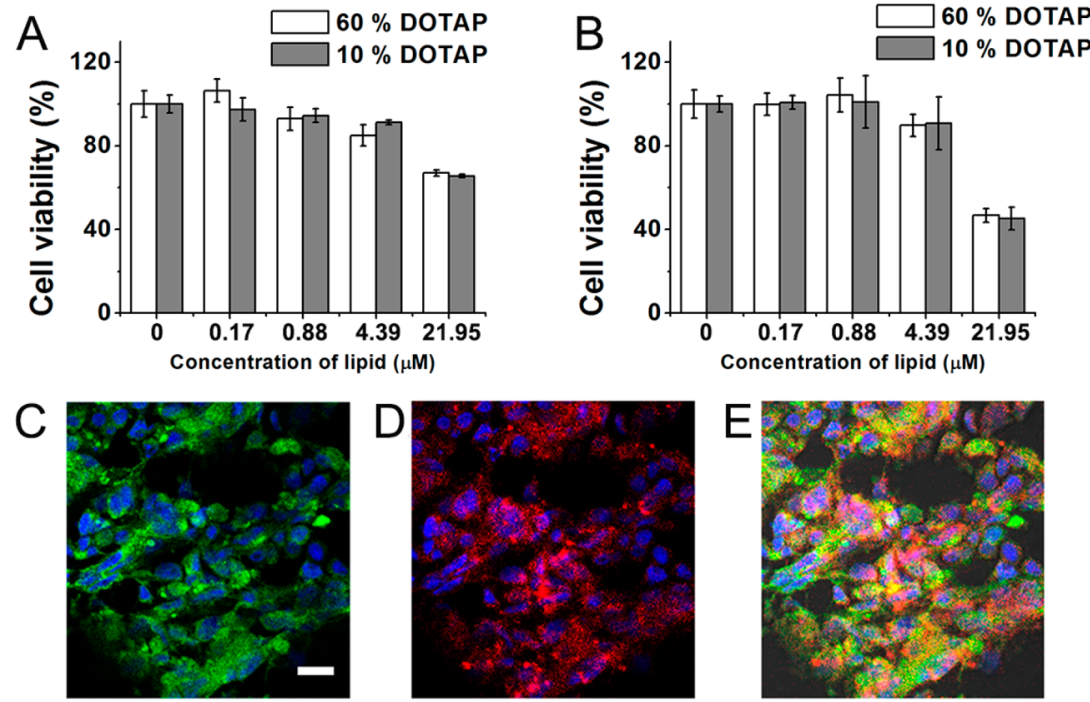

Figure 6. Cell viability of HUVEC cells after (A) $24 \mathrm{~h}$ and (B) $48 \mathrm{~h}$ incubation with nanoparticles with $10 \%$ and $60 \%$ DOTAP. (C, D) In vivo mitochondrial labeling using lipid-polymer nanoparticles with 60\% DOTAP and 20\% DOPE. Confocal microscopic images of the cryosection, in which the green fluorescence is from the immunohistochemistry-stained mitochondria, blue fluorescence from nucleus, and red fluorescence from DiD-labeled hybrid nanoparticles. (E) The overlaid images. The yellow fluorescence indicates the colocalization of hybrid nanoparticles and mitochondria. Scale bar, $20 \mu \mathrm{m}$.

fluorescence, Figure 6C) in the cryosection of tumor tissues, we find a good colocalization for nanoparticles and mitochondria (indicated by yellow areas in Figure $6 \mathrm{E}$ ). The calculated $R$ is $0.46 \pm 0.10$, higher than the threshold value (0.4) for statistically good correlation. ${ }^{47}$ Our results indicate the capability of hybrid nanoparticles with $60 \%$ DOTAP and $20 \%$ DOPE for both in vitro and in vivo mitochondria-specific distribution with good biocompatibility.

\section{CONCLUSION}

In summary, we present a study on the nonspecific organelletargeted distribution of lipid-polymer nanoparticles with the same size, core-shell structure but different lipid components/ ratios, which are precisely fabricated by a microfluidic device. A tendency from lysosomal-targeted to mitochondrial-targeted distribution of hybrid nanoparticles is revealed by increasing the DOTAP/DOPE ratio in the lipid shell. The functions of DOTAP and DOPE are systemically studied by experimental and numerical methods, suggesting an incremental effect of cationic and neutral helper lipids on lysosomal escape as well as mitochondrial targeting. This lipid-regulated subcellular distribution of nanoparticles could avoid the use of target moieties and complex synthetic steps, showing great promise for intracellular delivery of various drugs and enhancing their therapeutic efficacy.

\section{ASSOCIATED CONTENT}

\section{S Supporting Information}

The Supporting Information is available free of charge on the ACS Publications website at DOI: 10.1021/acs.analchem.6b01749.

Additional experimental data, including molar ratio of varied components in lipid solution; design of the twostage microfluidic chip; cellular uptake of hybrid nanoparticles; scatter plots generated by a plugin of ImageJ (PDF)

\section{AUTHOR INFORMATION}

\section{Corresponding Author}

*E-mail: sunjs@nanoctr.cn.

\section{Author Contributions}

The manuscript was written through contributions of all authors.

\section{Notes}

The authors declare no competing financial interest.

\section{ACKNOWLEDGMENTS}

We acknowledge financial support from MOST (2013AA032204), NSFC (21475028, 11422215, 11272327, and 11023001), and Youth Innovation Promotion Association CAS.

\section{REFERENCES}

(1) Torchilin, V. P. Annu. Rev. Biomed. Eng. 2006, 8, 343-375.

(2) Han, K.; Lei, Q.; Wang, S. B.; Hu, J. J.; Qiu, W. X.; Zhu, J. Y.; Yin,

W. N.; Luo, X.; Zhang, X. Z. Adv. Funct. Mater. 2015, 25, 2961-2971.

(3) Tian, J.; Ding, L.; Ju, H.; Yang, Y.; Li, X.; Shen, Z.; Zhu, Z.; Yu, J.

S.; Yang, C. J. Angew. Chem., Int. Ed. 2014, 53, 9544-9549.

(4) Costantini, P.; Jacotot, E.; Decaudin, D.; Kroemer, G. J. Natl. Cancer I 2000, 92, 1042-1053.

(5) Gregoriadis, G. Nature 1978, 275, 695-696.

(6) Shen, J.; Zhou, Y.; Lu, T.; Peng, J. Y.; Lin, Z. X.; Huang, L.; Pang,

Y. H.; Yu, L.; Huang, Y. Y. Lab Chip 2012, 12, 317-324.

(7) Xu, Z.; Liu, S.; Kang, Y.; Wang, M. Nanoscale 2015, 7, 58595868.

(8) Marrache, S.; Dhar, S. Proc. Natl. Acad. Sci. U. S. A. 2012, 109, $16288-16293$.

(9) Hu, Q. L.; Gao, M.; Feng, G. X.; Liu, B. Angew. Chem., Int. Ed. 2014, 53, 14225-14229.

(10) Jiang, L.; Li, L.; He, X.; Yi, Q.; He, B.; Cao, J.; Pan, W.; Gu, Z. Biomaterials 2015, 52, 126-139.

(11) Furukawa, R.; Yamada, Y.; Kawamura, E.; Harashima, H. Biomaterials 2015, 57, 107-115.

(12) Yang, L.; Li, N.; Pan, W.; Yu, Z.; Tang, B. Anal. Chem. 2015, 87, $3678-3684$. 
(13) Koshkaryev, A.; Thekkedath, R.; Pagano, C.; Meerovich, I.; Torchilin, V. P. J. Drug Targeting 2011, 19, 606-614.

(14) Wexselblatt, E.; Esko, J. D.; Tor, Y. ACS Nano 2015, 9, 39613968.

(15) Valencia, P. M.; Farokhzad, O. C.; Karnik, R.; Langer, R. Nat. Nanotechnol. 2012, 7, 623-629.

(16) Cheng, Z.; Al Zaki, A.; Hui, J. Z.; Muzykantov, V. R.; Tsourkas, A. Science 2012, 338, 903-910.

(17) Wang, T.; Wang, D.; Yu, H.; Wang, M.; Liu, J.; Feng, B.; Zhou, F.; Yin, Q.; Zhang, Z.; Huang, Y.; Li, Y. ACS Nano 2016, 10, 34963508.

(18) Hu, G.; Jiao, B.; Shi, X.; Valle, R. P.; Fan, Q.; Zuo, Y. Y. ACS Nano 2013, 7, 10525-10533.

(19) Yu, H. J.; Cui, Z. R.; Yu, P. C.; Guo, C. Y.; Feng, B.; Jiang, T. Y.; Wang, S. L.; Yin, Q.; Zhong, D. F.; Yang, X. L.; Zhang, Z. W.; Li, Y. P. Adv. Funct. Mater. 2015, 25, 2489-2500.

(20) Zeng, J. F.; Jing, L. H.; Hou, Y.; Jiao, M. X.; Qiao, R. R.; Jia, Q. J.; Liu, C. Y.; Fang, F.; Lei, H.; Gao, M. Y. Adv. Mater. 2014, 26, 26942698.

(21) Zhang, L.; Feng, Q.; Wang, J. L.; Zhang, S.; Ding, B. Q.; Wei, Y. J.; Dong, M. D.; Ryu, J. Y.; Yoon, T. Y.; Shi, X. H.; Sun, J. S.; Jiang, X. Y. ACS Nano 2015, 9, 9912-9921.

(22) Zhang, L.; Feng, Q.; Wang, J.; Sun, J.; Shi, X.; Jiang, X. Angew. Chem., Int. Ed. 2015, 54, 3952-3956.

(23) Zhang, Y.; Kim, W. Y.; Huang, L. Biomaterials 2013, 34, 34473458 .

(24) Chan, J. M.; Zhang, L.; Yuet, K. P.; Liao, G.; Rhee, J. W.; Langer, R.; Farokhzad, O. C. Biomaterials 2009, 30, 1627-1634.

(25) Feng, Q.; Yu, M. Z.; Wang, J. C.; Hou, W. J.; Gao, L. Y.; Ma, X. F.; Pei, X. W.; Niu, Y. J.; Liu, X. Y.; Qiu, C.; Pang, W. H.; Du, L. L.; Zhang, Q. Biomaterials 2014, 35, 5028-5038.

(26) Sun, J.; Zhang, L.; Wang, J.; Feng, Q.; Liu, D.; Yin, Q.; Xu, D.; Wei, Y.; Ding, B.; Shi, X.; Jiang, X. Adv. Mater. 2015, 27, 1402-1407.

(27) Zhang, S.; Gao, H.; Bao, G. ACS Nano 2015, 9, 8655-8671.

(28) Wang, J.; Chen, W.; Sun, J.; Liu, C.; Yin, Q.; Zhang, L.; Xianyu, Y.; Shi, X.; Hu, G.; Jiang, X. Lab Chip 2014, 14, 1673-1677.

(29) Sun, Y.; Yong, K. M.; Villa-Diaz, L. G.; Zhang, X.; Chen, W.; Philson, R.; Weng, S.; Xu, H.; Krebsbach, P. H.; Fu, J. Nat. Mater. 2014, 13, 599-604.

(30) Hartmann, R.; Weidenbach, M.; Neubauer, M.; Fery, A.; Parak, W. J. Angew. Chem., Int. Ed. 2015, 54, 1365-1368.

(31) Hafez, I. M.; Maurer, N.; Cullis, P. R. Gene Ther. 2001, 8, 11881196.

(32) Wang, H.; Di, J.; Sun, Y. B.; Fu, J. P.; Wei, Z. Y.; Matsui, H.; Alonso, A. D.; Zhou, S. Q. Adv. Funct. Mater. 2015, 25, 5537-5547.

(33) Fu, Y.; Li, C.; Lu, S.; Zhou, W.; Tang, F.; Xie, X. S.; Huang, Y. Proc. Natl. Acad. Sci. U. S. A. 2015, 112, 11923-11928.

(34) Liu, X.; Yi, Q.; Han, Y.; Liang, Z.; Shen, C.; Zhou, Z.; Sun, J. L.;

Li, Y.; Du, W.; Cao, R. Angew. Chem., Int. Ed. 2015, 54, 1846-1850.

(35) Han, Y. L.; Wang, W.; Hu, J.; Huang, G.; Wang, S.; Lee, W. G.; Lu, T. J.; Xu, F. Lab Chip 2013, 13, 4745-4749.

(36) Shi, M.; Majumdar, D.; Gao, Y.; Brewer, B. M.; Goodwin, C. R.; McLean, J. A.; Li, D.; Webb, D. J. Lab Chip 2013, 13, 3008-3021.

(37) Xu, P.; Zheng, X.; Tao, Y.; Du, W. Anal. Chem. 2016, 88, 3171.

(38) Wang, J. D.; Lu, W. J.; Tang, C. H.; Liu, Y.; Sun, J. S.; Mu, X.; Zhang, L.; Dai, B.; Li, X. Y.; Zhuo, H. L.; Jiang, X. Y. Anal. Chem. 2015, 87, 11893-11900.

(39) Lu, X.; Xuan, X. Anal. Chem. 2015, 87, 6389-6396.

(40) Yu, Z.; Lu, S.; Huang, Y. Anal. Chem. 2014, 86, 9386-9390.

(41) Zinchuk, V.; Zinchuk, O.; Okada, T. Acta Histochem. Cytochem.

2007, 40, 101-111.

(42) Hulme, S. E.; Shevkoplyas, S. S.; McGuigan, A. P.; Apfeld, J.; Fontana, W.; Whitesides, G. M. Lab Chip 2010, 10, 589-597.

(43) Varkouhi, A. K.; Scholte, M.; Storm, G.; Haisma, H. J. J. Controlled Release 2011, 151, 220-228.

(44) Qualls, M. M.; Thompson, D. H. Int. J. Cancer 2001, 93, 384392.

(45) Bouxsein, N. F.; McAllister, C. S.; Ewert, K. K.; Samuel, C. E.; Safinya, C. R. Biochemistry 2007, 46, 4785-4792.
(46) Marrink, S. J.; de Vries, A. H.; Mark, A. E. J. Phys. Chem. B 2004, $108,750-760$.

(47) Cardarelli, F.; Pozzi, D.; Bifone, A.; Marchini, C.; Caracciolo, G. Mol. Pharmaceutics 2012, 9, 334-340. 